\title{
Changes in Breathing Mode, Sensory Profile and Malocclusions in Infant Patients
}

\author{
Brígida SD Bem, Gabriela S Ferreira, Richelle NA Costa, Daniele A Cunha, Ana Carollyne D Lima, Hilton J \\ Silva, Leonardo CB Santos and Luciana BC Fontes* \\ Department of Clinical and Preventive Dentistry, Federal University of Pernambuco, Brazil
}

*Corresponding author: Luciana de Barros Correia Fontes, Department of Clinical and Preventive Dentistry, Federal University of Pernambuco, Brazil.

\section{Abstract}

Objective: To verify the frequency of respiratory mode changes among children assisted at a dental school, as well as the impact of this condition on the oral processing of the individual in question, comparing data recorded in medical records and those obtained in clinical and functional examination.

Method: Cross-sectional study with descriptive and inferential treatment of data, developed with children undergoing dental treatment at the Federal University of Pernambuco, Recife, northeast of Brazil and their mothers, during the first semester of 2019. These are in the age group of six to nine years. and in the mixed dentition phase, according to the defined inclusion and exclusion criteria. The project was approved by the UFPE Ethics Committee. For the statistical analysis a margin of error of $5 \%$ and the Fisher's exact teste were adopted. Medical records and clinical and functional evaluations were considered regarding breathing mode, sensory profile and malocclusions.

Results: For a universe of 208 medical records, 33 (15.9\%) had the record of respiratory alterations, with allergic rhinitis and asthma being the registered conditions. Among the children with respiratory alterations, $21.2 \%$ had mouth breathing, $18.2 \%$ had sleep alterations and $36.4 \%$ had malocclusions, with no reports of changes in sensory processing. After clinical examination and functional evaluation for the 33 children with respiratory disorders, $48.5 \%$ had sleep disorders, $97.0 \%$ had some sensory processing disorder and $81.8 \%$ had malocclusions, with significant differences between the records and the data obtained after the evaluations. The most frequent malocclusions were Angle Class I, open bite and unilateral crossbite.

Conclusion: There was a small record of respiratory changes in the records of children assisted by dentistry; however, most of these had malocclusions and disorders in sensory processing with implications for oral sensitivity.

Keywords: Malocclusion; Mouth breathing; Children; Sensation

\section{Introduction}

Respiratory problems have been a major challenge to global public health due to its high incidence and opportunity to impact the quality of life of affected individuals. Rising Earth surface temperatures, which often increase air pollution, bring the possibility of reduced lung function and the aggravation of breathing disorders. In addition, climate change leads to increased production of airborne allergens with increased asthmatic episodes, particularly among children and adolescents [1,2]. Regarding the influence of breathing on craniofacial morphology, there are several publications in the literature that have highlighted a relationship between nasal breathing mode and the normal pattern of craniofacial growth and development of teeth and occlusion. The researchers pointed out that, in the opposite condition, probable impairments would also occur in the body posture and in the sensory and cognitive performance of patients with mouth or oral breathing [3-5].

Children chronically breathing through the mouth may develop speech disorders, inadequate body posture, changes in the respiratory system, deformities of the face, and poor positioning of the teeth, leading to structural changes in the face, including lips, tongue, palate, and jaw, which will adapt to new breathing pattern. With this there is a buccolingual imbalance and consequently in the facial muscles, generating an important functional deficiency [6]. Despite evidence in the literature about the possible association between breathing mode and malocclusions, there is no further information on the frequency of respiratory problems among children attending dental school clinics, as well as the impact this condition in the oral processing of the individual concerned. The 
objective of the present study covers this investigation, highlighting whether there is a relationship between what is reported during the anamnesis, by those responsible, and what is found functionally.

\section{Material and Methods}

Cross-sectional study with descriptive and inferential data analysis. The study area included the Pediatric Outpatient Clinic of the University Hospital, Federal University of Pernambuco (UFPE) in Recife, the dental school-clinic of the same Higher Education Institution, the universe comprised all children assisted at the dental school clinic, in the first semester of 2019. As inclusion criteria, the sample included children from six to nine years old, with the history or record of some type of respiratory mode alteration in the anamnesis and in the mixed dentition phase, whose records included evaluations of Speech Therapy, Dentistry and Occupational Therapy. There was the exclusion of children, under speech therapy, otorhinolaryngology therapy or with the use of functional orthodontic or orthopedic appliances of the jaws. Also, those that presented some organic or neurological condition that made the communication or the functional assessment tests of the respiratory mode unfeasible.

The following study variables were age, gender, maternal level of education, presence and type of respiratory disorder, sleep problems, diagnosis of oral breathing, sensory profile, presence and classification of malocclusion. The assessment of the breathing mode was performed by otorhinolaryngology and speech pathology professionals, adopting the Protocol of Identification of Signs and Symptoms of Oral Breathing (PISSRO), according to the supplementary material, prepared by the Pathophysiology Research Group of the Stomatognathic System - GPPSE / UFPE, with information on breathing mode (with related signs and symptoms and nosologically diagnosis).

In the protocol cited above, the percentage distribution for functional diagnosis adds up to a total of responses and observations: less than $40 \%$ - no changes in breathing mode; $41 \%$ to $60 \%$-mild oral breathing mode; from $61 \%$ to $80 \%$-moderate breathing; above $80 \%$-severe oral breathing. For the analysis of sensory processing, performed by an occupational therapist, the Sensory Profile Test 2 was used. This instrument captures information regarding sensory processing (auditory, visual, vestibular, tactile, multisensory and oral sensory), sensory modulation (tolerance and tone, related to body position and movement, movement modulation affecting alert level, sensory modulation affecting emotional responses, modulation of visual stimulus affecting emotional response and activity level) and emotional and behavioral responses (behavioral and emotional responses, behavioral results of sensory processing, items indicating response thresholds). The data from this analysis included the participation of mothers in the responses.

The record of the presence and types of sleep and respiratory alterations, as well as the presence and type of malocclusion existed from the data in each patient's chart, establishing a new intraoral physical examination of these patients for occlusal evaluation [7].
This research respected the universal principles of bioethics and human rights. It was previously approved by the UFPE Ethics Committee. For data analysis, the Statistical Package for Social Sciences Software (SPSS - version 18) and Excel 2010 were used. The results are presented in table form, with their respective absolute and relative frequencies. To verify the existence of an association between oral breathing and the presence of malocclusion, Fisher's Exact Test was adopted. The 95\% confidence interval was adopted and only p values $<0.05$ were considered statistically significant.

\section{Results}

There was a survey of 208 medical records referring to child patients from six to nine years old, in attendance in the first half of 2019, for the dental school clinic in question. These included respiratory changes for 33 children, according to the inclusion and exclusion criteria; 17 were male $(51.5 \%)$ and had a mean age of $8( \pm$ $1.39)$ years. The average age of the mothers was $34( \pm 8.92)$ years and complete high school education (75.8\%).

Respiratory abnormalities were allergic in 14 children (42.4\% with allergic rhinitis) and asthma in 12 (36.4\%). Among the 33 patients in whom the type of respiratory disorder was discriminated, two (6.1\% reported asthma and allergy). For seven children $(21.2 \%)$ there was no specific cause. Regarding the classification of the child's breathing mode as oral breathing, seven were in this perspective (21.2\%). Six children had sleep problems (18.2\%) and 12 (36.4\%) had malocclusions. There was no record of sensory processing disorder.

The distribution of malocclusions was as follows, considering the Angle classification: five Class I, three Class II division 1 and one Class III. For the remaining four the type was not specified. Considering the registration of the other malocclusions: from the unilateral posterior crossbite records, two for the anterior open bite and two for the anterior crossbite. There was a record for bilateral posterior crossbite. Five children did not find the classification of malocclusion. Comparing the data obtained from the medical records with the respiratory mode assessments and the intraoral physical examinations of the patients, there were statistically significant differences $\mathrm{p}<0.05$ in relation to the patients with oral breathing (all patients registered with respiratory alterations had this type of mode, either in the functional evaluation or in the evaluation made by the otolaryngologist).

Concerning the presence of malocclusions; these were found in $27(81.8 \%)$ of the children assisted at the dental school in question after the intraoral physical examination, especially for Class I, Class II division 1, Class II, division II malocclusions and Class III. Also, for other types of malocclusions such as anterior open bite and unilateral posterior crossbite (Table 1). Although not included in the table, eight $(24.4 \%)$ of the evaluated children had primary crowding in the region of the lower permanent incisors. There was a significant difference between medical records and data from intraoral physical examination $(\mathrm{p}<0.05)$. Among the children evaluated, 16 (48.5\%) had sleep problems (Table 1). 
Table 1: Distribution of malocclusions evaluated after intraoral physical examination of children assisted at a dental clinic.

\begin{tabular}{|c|c|c|}
\hline Variable & N & $\%$ \\
\hline TOTAL & 33 & 100 \\
\hline Malocclusion & 27 & 81.8 \\
\hline \multicolumn{3}{|c|}{ Angle's Classification } \\
\hline Class I & 11 & 33.3 \\
\hline Class II (1 ${ }^{\text {st }}$ division) & 8 & 24.2 \\
\hline Class II (2 ${ }^{\text {nd }}$ division) & 5 & 15.2 \\
\hline Class III & 3 & 9.1 \\
\hline \multicolumn{2}{|c|}{ 0ther Classifications } \\
\hline Anterior open bite & 10 & 30.3 \\
\hline Severe overbite & 3 & 9.1 \\
\hline Anterior crossbite & 4 & 12.1 \\
\hline $\begin{array}{c}\text { Unilateral posterior } \\
\text { crossbite }\end{array}$ & 10 & 30.3 \\
\hline
\end{tabular}

Table 2 presents the distribution of children according to the presence and type of sensory alteration. According to their analysis, $32(97 \%)$ of the children with RO had some kind of alteration in the sensory profile, the most frequent being oral sensitivity and changes in the child's auditory processing and movement. When comparing patients' oral sensitivity to other sensory changes, there was a significant association between exploration, sensitivity, observation, socio-emotional and attention (Table 2).

Table 2: Distribution of oral breathing children analyzed according to the presence and type of change in sensory profile.

\begin{tabular}{|c|c|c|}
\hline Variable & N & \% \\
\hline TOTAL & 33 & 100 \\
\hline Presence & 27 & 81.8 \\
\hline Yes & 32 & 97 \\
\hline No & 1 & 3 \\
\hline Type & 33 & 100 \\
\hline Auditory & 16 & 48.5 \\
\hline Visual & 10 & 30.3 \\
\hline Tact & 9 & 27.3 \\
\hline Movement & 15 & 45.4 \\
\hline Body position & 12 & 36.4 \\
\hline Oral sensivity & 17 & 51.5 \\
\hline Conduct & 10 & 30.3 \\
\hline Socioemotional & 13 & 39.4 \\
\hline Attention & 13 & 39.4 \\
\hline
\end{tabular}

\section{Discussion}

One of the biggest challenges of the present study was the gathering of information in the dental records of the considered clinical school, due to information gaps. Clinical and functional examinations took place in only 33 assisted children. However, considering the differences observed between the recorded data and the clinical and functional exams, possibly changes in breathing mode may compromise a higher percentage of assisted children.

Dentistry students recognize the importance of completing the complete medical record8. However, during this research, some flaws in this sense were observed; especially after a new clinical and functional evaluation.

The dental record is a very important document for recording data of variables that guide the diagnosis and treatment plan. It has administrative, ethical, legal and legal aspects. Higher Education Institutions have a primary role in raising awareness of the need for complete and well-prepared dental documentation, with reinforcement in the training of future professionals [8].

Most orthodontists and functional orthopedists of the jaws form the diagnosis of breathing in the anamnesis phase, when one observes and/or questions about the harmful habits associated with a possibly altered breathing mode [9]. The early observation of the characteristics of the oral breathing mode performed by the dentist allows a diagnosis of sensory system disorders and malocclusions and also signals about possible behaviors or attitudes of the child when in the dental environment, which, it is usually full of stimuli for the human senses. The considered ideal breathing pattern can be replaced by the oral one, this functional adaptation can occur for organic and nonorganic causes (considered vicious). In organic breathing, there is nasal cavity obstruction due to chronic inflammation of the nasal mucosa, deviated septum, pharyngeal and / or palatine tonsil hypertrophy, polyps, tumors and others. Allergic rhinitis and increased adenoids and decreased or absent breastfeeding are still considered $[3,10,11]$.

Among the studies consulted in the literature, most authors agree that the most common implications of RO are changes in the craniofacial and dental systems, the phono articulatory, body, behavioral and oral functions. [2-4,11]. However, it was also mentioned that the methods to verify this relationship are not yet satisfactory. The lack of sample representativeness, followup over time and protocols validated for this purpose represent a challenge to be overcome $[12,13]$. In the present study, it was found that almost all children with oral breathing had some kind of sensory processing disorder, which can have a number of implications for the development of the individual, particularly in this very unique phase. The presence of frequent tiredness, daytime sleepiness, prostration, nocturnal enuresis, reduced appetite, nutritional alterations, poor cerebral oxygenation, learning deficit and impairments in some sensory systems, such as olfactory, taste and hearing, may cause speech and developmental difficulties. are listed during the literature reviews on changes in breathing mode $[10,12,14]$.

These changes in the Stomatognathic System (SS) and sensory integration could occur due to the organism adapting to the changes occurred by the new non-physiological breathing format, whether associated with organic, functional or neurological variables $[7,10,14]$. The oral breather patient does not respond correctly to external stimuli, with diminished or increased responses. Sensory processing plays an important role for the executive functions of the individual. Sensory integration is the neurological process that organizes the sensations of one's own body and environment; modulates the information received by the senses (taste, smell, 
sight, hearing, touch, movement, gravity and body position). When there is a disorder in this processing, it begins to generate an altered, diminished, or increased response to any stimulus, with a major impact on the performance of executive functions (skills that control our thoughts, emotions, and actions), on the performance of everyday activities, consequently on the quality of life $[10,14]$.

Among the types of alterations found in oral breathing children, in the current study, there was a higher frequency of changes in the processing of oral sensitivity, auditory perception and movement are closely related to the child's behavior and the conduct of the dentist, during the dental appointment. The response of oral breather in manipulating the face, specifically its oral cavity, will depend on how the dental surgeon will approach the individual in order to familiarize him not only with the environment but also with the treatment itself and together with other professional, giving the necessary support and seeking understanding of the whole context in which the oral breather is inserted, from a morpho functional problem to the psychosocial impact on the life of this individual. The most frequent orofacial and postural characteristics in the oral breather include: elongated face, narrow nostrils, inadequate lip seal, lack of facial muscle tone, slanted or slanted eyes, dark circles, shoulder closure, spinal imbalance, small nose, hypotonic lips , dry, everted, narrow and deep palate, open mouth, atresic upper arch, Class II, malocclusions (facial asymmetry, open bite and posterior cross) and alterations in swallowing, suction and phonation [2,3,11-15].

Changes in sleep and malocclusions were considered here, and according to the data obtained for the latter, about one third of the children assisted had Angle Class II, anterior open bite and unilateral posterior crossbite. This result is similar to that of the study consulted for Angle Class II division 1; but without significant association with breathing mode or facial type [16]. Subsequent intervention with functional orthodontic and orthopedic features of the jaws was performed to treat installed malocclusions. The need for prevention and early (interceptive) treatment of oral breathing is reinforced to avoid the development of malocclusions or increased complexity, with the worsening of the conditions that are present from the perspective of a multidisciplinary approach [17]. This research emphasizes the importance of the integrated performance of Dentistry, with professionals of Speech Therapy, Occupational Medicine and Therapy, besides the approach of Psychology, in the search for comprehensive and humanized assistance to children with RO, reducing negative repercussions, not only in the orofacial complex, but in the approach to the global development of these individuals.

\section{Conclusion}

There was a small record of respiratory changes in the records of children assisted by dentistry; however, most of these had malocclusions and disorders in sensory processing with implications for oral sensitivity.

Of the twelve domains evaluated for the relationship between sensory alterations and oral processing, the exploration, sensitivity, observation, socio-emotional and attention domains present a more significant association with patients who have oral breathing, suggesting a relationship between oral breathing and sensory processing disorders of these individuals.

\section{Acknowledgement}

None.

\section{Conflict of Interest}

No conflict of interest.

\section{References}

1. Burki TL (2016) What will COP21 mean for global respiratory health? Lancet Respir Med 4: 96.

2. Ribeiro GCA, Santos ID, Santos CAN, Paranhos LR, César CPHAR (2016) Influence of the breathing pattern on the learning process: a systematic review of literature. Braz j otorhinolaringol 82: 466-478.

3. Grippaudo C, Paolantonio EG, Antoini G, Saulle R, La Torre G, et al. (2016) Association between oral habits, mouth breathing and malocclusion. Acta Orothinolaryngol Ital 36: 386-394.

4. Imbaud TC, Mallozi MC, Domingos VBTC, Solé D (2016) Frequency of rhinits and orofacial disorders in patients with dental malocclusion. Rev paul pediatr 34(2): 184-188.

5. Silva RA, Jóias RM, Josgriberg E, Rode SM, Paranhos R, Jóias RP (2016) The correlation between malocclusions and morplho functional aspects: analysis of patients aged from 7 to 12 years old. Braz Dent Sci 19(4): 90-97.

6. Cunha DA, Silva GAP, Motta MEFA, Lima CR, Silva HJ (2007) Mouth breathing in children and its repercussions in the nutritional state. Rev CEFAC 9(1): 41-54.

7. Bittencourt MAV, Machado AW (2010) An overview of the prevalence of malocclusion in 6 to 10-year-old children in Brazil. Dental Press J Orthod 15:133-142.

8. Oliveira DL, Yarid SD (2014) Dental records from the perspective of dentistry students. Rev Odontol UNESP 43(3): 158-164.

9. Menezes VA, Cavalcanti LL, Albuquerque TC, Garcia AFG, Leal RB (2011) Mouth breathing within a multidisciplinary approach: Perception of orthodontists in the city of Recife, Brazil. Dental Press J Orthod 16(6): 84-92.

10. Neiva PD, Kirkwood RN, Mendes PL, Zabjek K, Becker HG, Mathur S (2018) Postural disorders in mouth breathing children: a systematic review. Braz J Phys Ther 22(1): 7-19.

11. Lima ACD, Cunha DA, Albuquerque RC, Costa RNA, Da Silva HJ (2019) Sensory changes in mouth breathers: systematic review based on the PRISMA method. Rev Paul Pediatr 37(1): 97-103.

12. Lessa FCR, Enoki C, Feres MFN, Valera FCP, Lima WTA, et al. (2005) Breathing mode influence in craniofacial development. Braz J Otorrinolaringol 71(2): 156-160.

13. Barbosa OLC, Gonçalves NM, Barbosa N, Silva FSC (2016) Respiratory type as a predisposing factor for the formation of malocclusion. Revista Pró UniverSUS 7(2): 17-21.

14. Santos MH, Dantas Filho MO, Barros Correia MC, Barros Correia CW, Souza Júnior VR, et al. (2018) Deleterious oral habits in preschool children with sensory processing disorder. Adv Dental \& Oral Health 9(3): 3-7.

15. Popoaski TFM, Sakae TM, Schmitz LM, Correa LHL (2012) Evaluation from the quality of life in the oral breathers' patients. Arq Int Otorrinolaringol 16(1): 74-81.

16. Nastri VHT, Bommarito S (2007) Breath evaluation based on different facial types in patients with malocclusion. Rev Odonto 15(30): 97-106.

17. Paolantonio EG, Ludovici N, Saccomanno S, La Torre G, Grippaudo C (2019) Association between oral habits, mouth breathing and malocclusion in italian preschoolers. Eur J Paediatr Dent 20(3): 204-208. 